\title{
Construyendo alianzas para formación continuada de docentes en la educación básica (LASEB): una experiencia brasileña
}

Building Alliances

for Continuing

Education of

Teachers in Basic

Education (LASEB): A

Brazilian Experience
Construindo alianças

para a Formação

Continuada de

Docentes do Ensino

básico (LASEB): uma

experiência brasileira

Maria de Fátima Cardoso Gomes*

Fecha de recepción: 12-01-2018

Fecha de aprobación: 3-07-2018

\section{PARA CITAR ESTE ARTÍCULO}

Cardoso, M. (2018). Construyendo alianzas para formación continuada de docentes en la educación básica (LASEB): una experiencia brasileña. Nodos y Nudos, 6(44), 47-58.
Coordinadora del Grupo de Estudios e Investigación en Psicologia Histórica cultural en el Aula. (GEPSA). Coordinadora del programa de posgrado en Educación: Conocimiento e inclusión social de la Facultad de Educación de la ufMg. mafacg@gmail.com 


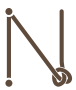

Volumen $5 \mathrm{~N} .{ }^{\circ} 44$ enero - junio de 2018 ISSN: 0122-4328 ISSN-E: 2619-6069 pp. 47-58

\section{RESUMEN}

Este trabajo pretende discutir sobre la experiencia de formación continuada de docentes en la educación básica (LASEB) y la construcción de estrategias de trabajo en equipo entre la Facultad de Educación de la Universidad Federal de Minas Gerais y la Secretaría Municipal de Educación de Belo Horizonte, Minas Gerais (Brasil), del 2006 al 2014. EL programa tiene por objetivo mejorar la enseñanza y la calidad en la educación de diferentes alumnos de diversas áreas como: Alfabetización, Matemáticas, Educación Infantil, Juventud y Escuela, Historia del África y Cultura Afrobrasilera, Cine y Educación, Ciencias y la Comprensión de los Procesos de Enseñanza, y Aprendizaje en la Educación Básica. En dichas áreas pretendemos favorecer el desarrollo de estudios y actividades que posibiliten una estrecha articulación entre el debate teórico-epistemológico y la práctica de los docentes en el cotidiano de la escuela y la sala de aula. El impacto que tuvieron los planes de acción, elaborados durante la formación teórica y práctica de los profesores en el curso y que eran aplicados en las escuelas y salas de aula, es fundamental en esta reflexión. Vale decir que los planes de acción constituyen para nosotros mediadores semióticos que posibilitan los avances, retrocesos y la resignificación de sus prácticas pedagógicas. Pues, para nosotros educar es más que un proceso formal de escolarización y debe ser considerado como una política estratégica de desarrollo e inclusión social.

Palabras clave: formación continuada; docentes; educación básica; psicología histórico-cultural

\section{ABSTRACT}

This paper aims to discuss the experience of continuing education of teachers in basic education (LASEB) and the construction of teamwork strategies between the School of Education of Universidade Federal de Minas Gerais and the Municipal Secretary of Education of Belo Horizonte, Minas Gerais (Brazil), from 2006 to 2014. The goal of the program is to improve teaching and the quality of education of different students in several areas, such as Literacy, Mathematics, Early Childhood Education, Youth and School, History of Africa and AfroBrazilian Culture, Film and Education, Science and Understanding of Teaching Processes, and Learning in Basic Education. In these areas, we intend to favor the development of studies and activities that allow a close articulation between the theoretical-epistemological debate and the practice of teachers in the daily life of the school and the classroom. The impact of the action plans developed during the theoretical and practical training of teachers in the course and which were applied in schools and classrooms, is fundamental in this reflection. It is worth noting that, to us, actions plans are semiotic mediators that enable the advances, setbacks, and the resignification of their pedagogical practices, since it is our belief that education is more than a formal process of schooling and that it should be considered as a strategic policy of development and social inclusion.

Keywords: continuing education; teachers; basic education; historical and cultural psychology 


\section{RESUMO}

Este trabajo pretende discutir sobre a experiencia de formação continuada de docentes na educación básica (LASEB) e a construção de estratégias de trabalho en equipe entre a Faculdade de Educação da Universidade Federal de Minas Gerais e a Secretaria Municipal de Educação de Belo Horizonte, Minas Gerais, Brasil, de 2006 até 2014. Este programa tem como objetivo melhorar o ensino e qualidade da educação de diferentes alunos de diversas áreas como: Alfabetização, Matemática, Educação Infantil, Juventude e Escola, Historia da África e Cultura Afrobrasileira, Cinema e Educação, Ciências e a Comprensão dos Processos de Ensino e Aprendizagem na Educação Básica. Em todas essas áreas pretendemos favorecer 0 desenvolvimento de estudos e atividades que posibilitem uma estreita articulação entre o debate teórico- epistemológico e a prática dos docentes no cotidiano da escola e da sala de aula. 0 impacto que tiveram os Planos de Acção, elaborados durante a formação teórica e prática dos profesores no curso e que foram aplicados nas escolas e salas de aula são fundamentais para esta reflexão. Vale dizer que os Planos de Ação são para nós mediadores semióticos que possibilitaram os avanços, retrocessos e a ressignificação de suas práticas pedagógicas. Pois, para nós educar es mais do que um processo formal de escolarização e deve ser considerado como uma política estratégica de desenvolvimento e inclusão social.

Palavras-chave: formação continuada; docentes; educação básica; psicologia histórico-cultural

\section{Introducción}

Nuestra experiencia como formadores de docentes para la Educación Básica en Brasil se confunde en medio de la lucha de muchos educadores brasileños que trabajan en pro de mejorar la calidad de la educación en las escuelas públicas de este país. Una de esas luchas hace referencia a las movilizaciones en favor de la formación continuada de docentes que, a pesar de actuar hace varios años en las aulas, no habian tenido la posibilidad de hacer un curso de especialización lato sensu. Con el objetivo de llegar a ese público, se firma el convenio -Facultad de Educación de la Universidad Federal de Minas Gerais (faE) UFMG) y Secretaría Municipal de Educación de la Alcaldía de Belo Horizonte (smed/PBH) en 2006, construyendo un programa de formación continuada para los docentes de la red municipal de educación de Belo Horizonte, Minas Gerais (Brasil)-, que según Almeida e Silva y Evaristo (2009) busca atender las demandas de la red en torno a
[...] atención de las demandas para mejorar los procesos de enseñanza y aprendizaje en la Red Municipal de Educación, mejorar el trabajo pedagógico con el educando, considerando las discusiones socioculturales de la contemporaneidad aliadas a intencionalidades pedagógicas de los profesores y necesidades educativas de los nuevos alumnos. (p. 11).

Después de muchas negociaciones entre la FAE/ UFMG y la SMED/PBH elaboramos en 2006 la propuesta del curso de posgraduación: Especialización en Docencia para la Educación Básica (LASEB) que pretendió abarcar inicialmente cuatro áreas de concentración: Alfabetización, Educación Matemática, Historia de África y Culturas Afrobrasileñas, y Juventud y Escuela, ya en 2008 se incluyó el área de educación infantil. En 2011 se dio una primera reconfiguración de las áreas, manteniendo algunas y sustituyendo otras: Alfabetización, Aprendizaje y Enseñanza en la Educación Básica, Educación de las Relaciones Étnico-Raciales, Educación Infantil y 
Educación Matemática. En 2013 se realizó de nuevo una reconfiguración de las áreas: Proceso de Alfabetización, Procesos de Aprendizaje y Enseñanza en la Educación Básica, Diversidad Étnica Racial y de Género, Múltiples Lenguajes en la Educación Infantil, Educación en Ciencias y Educación, y Cinema. Esos cambios se hacian con el objetivo de atender las demandas más urgentes de la Red Municipal de Educación de Belo Horizonte y las exigencias de la Prorrectoría de Posgraduación de la UFMG.

Además, ampliamos la propuesta de formación tanto para docentes como para coordinadores y directores de las escuelas de dicha red pública de enseñanza. Completamos ya 6 ediciones de este programa de formación de 2006 a 2014. En este momento nos encontramos en proceso de negociación con otras redes públicas de enseñanza de Belo Horizonte y otros municipios, pues la Red Municipal de Educación de Belo Horizonte no puede dar continuidad a esa formación por falta de presupuesto. Se hace necesario aclarar que todos los costos de este programa de formación son responsabilidad de los dirigentes de la Red de Educación de Belo Horizonte con el apoyo de recurso del Fondo Nacional de Desenvolvimiento de la Educación (FNDE), es decir, formación sin cobros económicos para los docentes, que llegaron a ser 1240, considerando las 6 ediciones de este programa de formación.

Una primera versión de este artículo fue presentado en el panel "Evaluación de maestros(as) y formación continuada", durante el Encuentro de Rectores(as) y Directivos Académicos(as), realizado en la Universidad Pedagógica Nacional de Colombia, el 6 de octubre de 2015. Luego de esta presentación se construyó este texto con el objetivo de ampliar y profundizar las ideas discutidas.

\section{LASEB: presupuestos teórico-metodológicos y estructura curricular}

La propuesta de LASEB se fundamentó en el acumulado conceptual y metodológico ya desarrollado y lo que se construyó en los Núcleos de Estudios e Investigación de la Facultad de Educación/UFMG, en especial en el Centro de Alfabetización, Lectura y Escritura (CEALE), por el Núcleo de Educación Matemática, el Grupo de
Acciones Afirmativas, el Observatorio de la Juventud y por el Núcleo de Educación Infantil. Además, la propuesta contó con una base sólida: la experiencia de trabajos anteriores desarrollados con la Secretaría Municipal de Educación de la Alcaldía de Belo Horizonte, en una larga historia de convenios (Gomes, Dalben, Rocha y Alves, 2009).

Por parte de la Secretaría Municipal de Educación habia un documento en elaboración en 2005 que explicaba algunas de las directrices para la construcción de la propuesta del LASEB:

Trabajar permanentemente, con problemas, desafíos 0 preguntas presentes en el día a día escolar y en la práctica docente; tratar los contenidos disciplinares y académico-científicos por medio de problemas, temáticas o de desafíos, en vez de ser desarrollados por medio de disciplinas o contenidos disciplinares estancados y descontextualizados. (PBH/SMED, 2005).

Por otro lado, la Facultad de Educación de la Universidad Federal de Minas Gerais buscó poner en práctica sus principios básicos de formación docente, perspectiva desde la cual, surgieron los principios orientadores de la formación LASEB:

a. Valorización de la experiencia profesional de los profesores.

b. Reflexión sobre esa experiencia con una perspectiva de toma de conciencia y de distanciamiento crítico necesario para que se torne objeto de investigación.

c. Énfasis en la pluralidad y en el respeto a la identidad cultural de los sujetos a quien se destina esta formación -profesores y alumnos-.

d. Uso de diferentes tecnologías de formación en la perspectiva de ampliar horizontes teórico-metodológicos de producción de conocimiento.

e. Reflexión sobre la realidad actual de la educación en el municipio, en el Estado y en el país, así como en el análisis de los problemas educativos mundiales.

f. Reflexión sobre cuestiones contemporáneas pertinentes a la producción de conocimiento y a las fronteras entre las disciplinas, previniendo 
espacios y tiempos para la articulación entre áreas y disciplinas.

g. Énfasis en la construcción y reconstrucción de la identidad profesional del docente ante el contexto contemporáneo. (Gomes et al., 2009, p. 21).

Hubo un concepto básico con el cual trabajamos que nos ayudó mucho en la comprensión del papel que la mediación y el uso de los Planes de Acción tenían como mediadores semióticos en la formación de los profesores del LASEB: la mediación semiótica. Para esto, tomamos como base la perspectiva que al respecto ofrece la psicología histórico-cultural (Vygotsky, 1993; 1995), que considera el conocimiento como el resultado de la actividad humana, construida esencialmente por instrumentos y signos mediadores, siendo así social, instrumental y productora. Ser social significa que además de ser socialmente planeada, tanto los instrumentos usados para su planeación, como el producto obtenido, pueden ser socializados, o sea, pueden ser usados por los otros, lo que según Pino (1995) "es un dato nuevo en la historia de la evolución" (p.31). De esta manera, "que la actividad sea instrumental significa dos cosas: que ella es siempre mediada por instrumentos y que estos son creados por los hombres en función de la naturaleza de las acciones planeadas por ellos" (p. 31). Los instrumentos son técnicos y semióticos; los primeros sirven para actuar sobre la naturaleza de los semióticos (signos) para producir comunicación, representación y significación de la realidad. Pino (1995) también explica que la idea de asuntos técnicos-instrumentales tiene origen en el marxismo mientras que la de semiótica es una innovación de Vygotsky (1993; 1995). Esos dos tipos de instrumentos presentan cosas en común; por ejemplo, "su función mediadora en las relaciones de los hombre entre sí y con el mundo" (Pino, 1995, p. 31). Este autor también plantea que "las caracteristicas instrumental y social de la actividad humana le confieren su cualidad de productora, es decir, de posibilitar la transformación simultanea del objeto y de los sujetos de acción" (p. 32). Esto significa la subjetivación de la objetivación, el producto de la acción puede ser reapropiado por el sujeto, y también la objetivación de la subjetivación, el producto de la acción, es la materialización de las cualidades del sujeto agente. Esto reubica al hombre en el orden de la cultura, más allá del orden biológico (Pino, 1995). Ese proceso de reapropiación del sujeto implica tornar como propio algo que antes no le pertenecía, y también implica hacer y usar instrumentos, en el sentido de la transformación de sí mismo, de los otros y de sus acciones en sus trabajos y producciones culturales.

En nuestras reflexiones nos interesó analizar el papel de la mediación semiótica en la formación de profesores, pues ella les confiere a profesores, formadores y en formación, la capacidad de reconocer las realidades educativas que vivenciaron en el plano de lo simbólico, al recurrir a sistemas de signos, particularmente lingüisticos, esto es, cuando podían decir, pensar y reflexionar sobre sus acciones en las escuelas y en las salas de aula, compartiendo con los otros, afectar y dejarse afectar por las experiencias, transformando a los otros y siendo transformado por esas acciones durante al formación en LASEB.

Una experiencia basada en estos argumentos podrá proporcionar el diálogo "en tres dimensiones -en formación, los ya formados y los gestores- con la oportunidad del reconocimiento mutuo de las necesidades, demandas y urgencias relativas a la práctica educativa que sucede en el día a día de la Educación Básica" (Gomes et al., 2009, p. 22).

Para evitar ausentismos, la propuesta LASEB fue implementada principalmente los sábados, 8 horas de actividades docentes, con algunos encuentros los viernes en la noche para actividades de estudio e investigación en la biblioteca y en los laboratorios de informática de la Facultad de Educación de la Universidad Federal de Minas Gerais (Brasil). Evitando el ausentismo, que es uno de los mayores problemas que se presenta cuando el profesor se ausenta para realizar sus cursos de formación, pues este genera sobrecarga de trabajo para otros docentes, presiones económicas y sociales, doble jornada de trabajo por la inexistencia de docentes disponibles para remplazarlos.

La estructura curricular del LASEB tiene una base común, integrada en la idea de un programa que se ramifica por medio de disciplinas específicas a cada área de concentración. La carga horaria total es de 450 
horas, entre dos disciplinas comunes de 60 horas, denominadas "Núcleo Común" y 11 disciplinas específicas de 30 horas, de diferente área de concentración. Entre las disciplinas especificas 3 eran comunes a todas las áreas de concentración, con vistas a la aglutinación de temáticas referentes a la formación general del profesor: plan de estudios, teoría y práctica, sociedad, cultura y educación; investigación e intervención en las escuelas.

Las disciplinas de Núcleo Común fueron estructuradas en torno a seminarios, palestras, talleres y diferentes grupos de reflexión sobre la práctica, ofrecidos a todos los estudiantes colectivamente. El objetivo fue propiciar un espacio en el que los docentes tuvieran la posibilidad de enfrentar los problemas relativos al aula de forma crítica, consciente y comprometida con el desarrollo humano y con cuestiones politicas y sociales más amplias, como la diversidad sociocultural de la sociedad en que nuestros alumnos están inmersos.

Según Gomes et al. (2009),

[...] al mismo tiempo que los seminarios y palestras contribuyeron a el debate referente a las áreas de concentración, con la presentación de resultados de investigaciones y estudios realizados sobre la realidad educativa de nuestro pais, los talleres le ofrecieron al profesor tiempos e espacios más específicos, para la reflexión relativa al que hacer en sala de aula. (p. 24).

De la misma manera, la otra disciplina de este núcleo común -"Análisis Critico de la Práctica Pedagógica (ACPP)- tiene como objetivo la formación y el acompañamiento más cercano de la práctica cotidiana de los docentes - los estudiantes fueron divididos en grupos de 10, cada uno con un tutor que orientaba las reflexiones sobre sus prácticas cotidianas en la escuela y en la sala de aula- y la elaboración de planes de acción en las escuelas o en las aulas como trabajos de fin de curso. Se pretendió, por tanto, instrumentalizar el profesor para que fuera capaz de reflexionar sobre su quehacer, sobre los procesos de evaluación de su trabajo, sobre los procesos de enseñanza/aprendizaje de sus alumnos, y también organizara, sistematizara actividades y elaborara planes de aula y proyectos de acción docente (Gomes et al., 2009).
De esta forma, pensamos que la elaboración de Planes de Acción y no de monografías como trabajo final de curso, debería permitir

[...] que las diferentes dimensiones de formación docente propuestas por el curso sean transformadoras en procesos concretos de reflexión/acción y producción de conocimientos sobre la enseñanza, la escuela y la práctica pedagógica vivenciada por los profesores en sus salas de aula y escuelas. (Gomes et al., 2009, pp. 25-26).

\section{Método de acción/reflexión. Acción del LASEB}

Al definir la práctica escolar como foco de nuestra formación de docentes en $\angle A S E B$, deseábamos ofrecer a los profesores en formación la lectura, el estudio y el análisis teórico de esa práctica, sistematizando, a lo largo del curso, la comprensión teórica, epistemológica y metodológica de dicha práctica. Otra de nuestras preocupaciones fue con la dimensión personal de la formación de esos docentes; perspectiva que está relacionada con la historia de desvalorización continua, que ha venido atravesando la profesión en Brasil. De esta manera, nuestras aulas/disciplinas específicas, seminarios, talleres y ACPP, debian ser muy bien planeadas, con contenidos interesantes y actuales, vivencias prácticas y teóricas capaces de alterar la práctica actual en las escuelas de la red municipal de educación de Belo Horizonte (Gomes et al., 2009).

Para que pudiéramos generar cambios en las prácticas de los docentes tomamos como eje de nuestro trabajo a la disciplina ACPP, novedad para los cursos de especialización. No obstante en nivel de maestria ya veníamos usando la reflexión sobre las experiencias de los estudiantes de maestría en sus escuelas y salas de aula como punto de partida para el delineamiento de sus objetos de estudio. En el caso de LASEB, la ACPP debería incentivar reflexiones colectivas profundas sobre la práctica educativa de grupos de profesores que con la orientación de un tutor reflexionaban y formulaban los planes de acción para aplicar en sus escuelas y salas de aula.

En ese sentido, nuestro deseo fue promover la formación de docentes inquietos, que cuestionan sus prácticas, buscan, investigan, crean y enseñan. Además de "favorecer la movilización frente a cuestiones 
relevantes que están presentes en la construcción de políticas públicas a partir de un conocimiento más profundo sobre sus procesos de elaboración y gestión" (Dalben, 2009, p. 321).

\section{Participantes de la formación continuada del LASEB}

Los docentes que participaron de las 6 ediciones del LASEB obedecieron a la siguiente distribución: los grupos de cada área de concentración contaban con 40 alumnos/docentes, $10 \%$ de los cupos fueron reservadas para profesores con discapacidad; $40 \%$ de los cupos para profesores que se declararan negros; y $50 \%$ de los cupos, para la categoría "otros". Además, ellos debian tener estabilidad laboral en las escuelas a las que pertenecían; actuar en sala de clase o en la coordinación pedagógica; no ocupar cargos de comisiones; haber cumplido cinco años en el magisterio; haber concluido curso de pregrado en licenciatura en cualquier área del conocimiento o en pedagogía; contar con disponibilidad de tiempo para sus estudios y cumplir las exigencias del curso; no haber concluido o estar cursando posgrado lato sensu o estricto sensu en cualquier área del conocimiento, asumir la responsabilidad de permanecer en la misma escuela o unidad de educación infantil durante el curso y después de su conclusión por mínimo dos años (Gomes et al., 2009).

\section{Resultados y discusión}

Teniendo en cuenta aquellos fines y metodología de acción y reflexión, elaboramos un cuestionario a finales de 2006, para hacer una evaluación del LASEB por los docentes/estudiantes; instrumento elaborado con base en la escala Lickert. Los docentes deberían manifestar su nivel de aprobación a frases sugeridas de forma afirmativa, positiva y propositiva. Frente a cada enunciado, el evaluado debía analizar y responder si estaba parcialmente de acuerdo, totalmente de acuerdo con la afirmación, o en algunos casos, si estaba en contra o si no podría evaluar la situación. Por tanto, el cuestionario,

[...] fue dividido en 5 partes, con afirmaciones que hacian referencia a: 1. Curso realizado; 2. Relación pedagógica que se construyó con el estudiante de la red municipal a partir del curso; 3. Organización del proceso de enseñanza;
4. Evaluación de las disciplinas del curso; 5. Coordinación del curso. (Dalben, 2009, p. 322).

En las siguientes ediciones, este instrumento fue modificado, pero para este artículo se hará referencia a las dos primeras ediciones del LASEB. El objetivo de esa evaluación fue conocer los impactos del curso en la vida personal y profesional de los docentes.

De acuerdo con Dalben (2009), en el primer bloque de preguntas pretendimos evaluar junto con los profesores si hubo cambios en su manera de interactuar con sus escuelas y colegas de trabajo, en lo que se refiere a la gestión de la escuela, el trabajo colectivo, a la ejecución de proyectos integrados, a su forma de presentarse ante el grupo:

Se observó que el $30 \%$ de los docentes respondieron que el curso aportó plenamente a su interacción con las cuestiones de gestión escolar, el 50 \% respondió que el curso les aportó solo parcialmente a su participación en la gestión general de la escuela. También el $80 \%$ consideraron que mejoró mucho su trabajo con los colegas y solo el $10 \%$ consideró que no podian hacer una evaluación de este tema. Todos los docentes que contestaron al cuestionario consideraron que ahora preparan mejor sus clases, leyendo periódicos y revistas con más frecuencia y utilizando esta información en sala de aula. De la misma manera, todos respondieron que después del curso están más interesados en asistir a conferencias, participar en eventos y congresos. La mayoria (80 \%) consideró que el curso favoreció plenamente el establecimiento de una relación pedagógica más efectiva y motivadora con los alumnos, y los demás estuvieron parcialmente de acuerdo con esta afirmación, sin haber ningún desacuerdo en este tema. (Dalben, 2009, pp. 322-323).

Continuando con la evaluación,

[...] el $80 \%$ de los estudiantes consideró que el curso aportó plenamente al fortalecimiento de la actitud y autoconfianza, frente a sus colegas, para manifestarse y presentar sus opiniones personales y dificultades. El otro $20 \%$ estuvo de acuerdo parcialmente con esta afirmación, indicando que se encuentran en un proceso de crecimiento personal que les permitirá tener más confianza y seguridad frente al grupo. (Dalben, 2009, p. 323).

Además, según Dalben (2009), en lo que tiene a ver con la relación pedagógica con los alumnos de la Red Municipal, cerca del $60 \%$ de los docentes 
afirmaron que sus alumnos estaban avanzando mucho más, participando más en las aulas, con mejores percepción en cuanto a sus avances y sus dificultades. Mientras que los demás encontraron que esto solo ocurría parcialmente.

La relación pedagógica fue un eje metodológico que impregnó, inclusive, las relaciones entre los profesores formadores, coordinadores y el equipo de la secretaría del LASEB y los docentes/estudiantes. Todos eran orientados para estar atentos a las demandas de los estudiantes docentes, sus experiencias y sus propuestas de acción. Había reuniones semanales del equipo de coordinadores de las áreas específicas y la secretaria del LASEB para atender las demandas y necesidades específicas de los estudiantes. Esto se hacía ya que muchas de las dificultades y angustias de los docentes/estudiantes podian ser solucionadas colectivamente por los coordinadores de las diferentes áreas, coordinación general y secretaía del curso. Esas dificultades y angustias causaron muchos conflictos que son propios de las relaciones interpersonales y que pasaron a ser un contenido para ser tratado en el aula, como punto de reflexión para todos nosotros, al punto de ser usados en las cuatro ediciones del LASEB.

En relación a las actividades en clase, el énfasis estuvo en la

[...] concepción de un profesor que debe ser cuidadoso para tener una sala de aula dinámica, que planea y selecciona actividades que le permitan observar las cualidades y caracteristicas de sus alumnos para conocerlo mejor, que abre un espacio para que sus estudiantes se expresen, que avalúa diferentes dimensiones y formatos, que utiliza en la sala de aula diferentes técnicas y soportes para la enseñanza y el aprendizaje y que emplea en la sala de aula de su escuela, lo que fue vivenciado en el curso. (Dalben, 2009, p. 324).

Las respuestas se dividieron entre los que estaban de acuerdo plenamente y los que solo lo hacian parcialmente, lo que indicó que la mayoría intentaba aplicar los conceptos estudiados modificando sus prácticas pedagógicas.

Sobre las disciplinas del LASEB, las respuestas estaban entre la atención plena y parcial, sin presentar problemas específicos en esa dimensión. Después de cada disciplina, los docentes/estudiantes respondian también un cuestionario de evaluación de los trabajos realizados por los diferentes profesores formadores y formulaban opiniones sobre posibles lagunas o puntos negativos en cada disciplina. Fueron evaluados más de 50 cuestionarios aleatoriamente seleccionados, de las dos primeras ediciones de LASEB que envolvieron en total más de 300 estudiantes.

A continuación, y de nuevo tomando como referencia a Dalben (2009) presentamos algunos testimonios que nos presentan su opinión sobre el curso de modo general y la oportunidad de formación ofrecida por la SMED/PBH, sobre las reglas pedagógicas, sobre la propia experiencia como estudiantes y como profesionales, sobre el proceso de evaluación del curso, sobre las actividades de enseñanza propuestos en el curso, y visiones negativas de esta experiencia en el LASEB, respectivamente:

Ese curso fue la mejor idea que a SMED ha tenido. La realidad de dar 60 o 70 aulas es muy pesada, y usted entra en una rutina, que hasta para de pensar. Hoy analizo mi práctica y reveo lo que hice y lo que hago. Veo que ya creé cosas y que adoro la matemática. Cuando comencé, "parecia que ia extrair o siso" (expresión brasileña que indica que el proceso es doloroso y dificil). Hoy estoy demasiado feliz. (Estudiante 1).

Hay varios puntos positivos en relación al curso y a los profesores: la excelente calidad de las aulas dadas; el envolvimiento y la competencia presentada por la mayoría de ellos; la preocupación con nuestra practica escolar, relacionado siempre la teoría y su aplicación efectiva en la escuela; el interés de los profesores por las dificultades presentadas por nuestros alumnos y por nosotros mismos, intentando ayudarnos siempre en la búsqueda de alternativas para el aprendizaje. (Estudiante 2).

Fue difícil cuando algunos profesores trabajaban contenidos intentando hacernos llegar hacia ellos, pero dejaban de observar las lagunas que teníamos. Muchas veces eran muy académicos. (Estudiante 3).

Siendo otra vez estudiante, percibí cuán fuerte es la influencia de la perspectiva del profesor sobre el alumno. Esa perspectiva puede hacerlo crecer o... (Estudiante 4).

El curso de posgrado propició un proceso evaluativo que posibilitó a los estudiantes cuestionarse sobre los principios de una evaluación tradicional. (Estudiante 5). 
Aprendi mucho, en el sentido de tener una mirada académica sobre las producciones de nuestros alumnos, esto es, el análisis de la práctica con base teórica, pues fui forzada a leer mucho para escribir mi proyecto - plan de acción. (Estudiante 6).

Algunos testimonios sobre la formulación de la propuesta de enseñanza (plan de acción) manifestaron las dificultades de algunos estudiantes, tal como mostramos a continuación, según el análisis de Dalben (2009):

Tuve todas las dificultades posibles. Desde aprender cómo darle formato al proyecto, hasta el contacto con los profesores. Hubo mucha confusión, y me era muy dificil trabajar de esa manera. No me senti estimulada con el trabajo y no tuve el retorno que necesite. El preproyecto que fue entregado de afán y que fue devuelto lleno de comentarios fue el inicio de mi desestímulo. (Estudiante 7).

En relación al proyecto de aprendizaje (plan de acción), sentí muchas dificultades para relacionar teoria y práctica y quise más orientación en el momento de buscar la teoría que aplicara al proyecto que respondiera mis dudas. (Estudiante 8).

\section{Conclusión}

Frente a estos testimonios podemos percibir el encuentro de experiencias desde el punto de vista como profesores de la educación básica y, al mismo tiempo, como estudiantes del LASEB, lo que posibilitó reflexionar sobre la propia práctica en las escuelas y aulas, y la propia formación como especialistas en las diferentes áreas de concentración. Reflexiones sobre los avances y dificultades, algunas superadas, otras en proceso de superación durante y después de la conclusión del curso. También por medio de los testimonios se puede deducir que la formación continuada de docentes no es algo simple, envuelve dimensiones personales, profesionales, político pedagógicas, institucionales, culturales e ideológicas. Dimensiones que pueden facilitar o impedir el desarrollo y aprendizaje de esos docentes.

Después de vivir la experiencia de 6 ediciones del LASEB logramos percibir una tendencia de los docentes/ estudiantes de particularizar sus prácticas y centrar sus intereses y esfuerzos en problemas muy especificos de su área de actuación, perdiendo la noción de conjunto del proceso de escolarización. Para nosotros, esto está vinculado a la falta de comprensión "del acto educativo como un acto político, y, por consiguiente, reflejo de las políticas públicas que, con la desvalorización de la profesión docente, le retiran la verdadera responsabilidad y compromiso social que le son exigidos" (Dalben y Gomes, 2012, p. 12). En este fenómeno sucede también con profesores de educación superior lo que nos lleva a pensar como Freire (1996), "desde el comienzo del proceso, va quedando cada vez más claro que, aunque diferentes entre sí, quien forma se forma y reforma, y quien es formado se forma y forma al ser formado" (p. 25).

De esta forma nuestro objetivo fue "sembrar la actitud reflexiva en la identidad profesional de profesores" (Perrenoud, 1999, p. 11) para que de ellos surgieran iniciativas colectivas e individuales en la solución de los problemas que se enfrentan día a día en el aula.

También nos fue posible experimentar el proceso de mediación semiótica (Vygotsky, 1993) pues, en la escritura de los planes de acción para las escuelas y salas de aula, les fue posible tomar distancia de sus prácticas, construir la capacidad de meta cognición y reinventar sus prácticas pedagógicas. Así, los docentes/estudiantes pudieron identificar problemas y buscar sus soluciones colectiva e individualmente, y con ello re-significaron sus prácticas pedagógicas, en el sentido de construcción de prácticas planeadas y sistemáticas que exploraran las diferencias sociales y culturales entre los alumnos, como factor fundamental para respetar los deseos y sentidos construidos por lo que se enseña y aprende en las escuelas públicas brasileras. Tenemos que reconocer de un lado, el papel de la institución escuela/LASEB en la construcción de los procesos psicológicos y sociohistórico-culturales de los profesores (Dalben y Gomes, 2012, p. 13). Por otro lado, de acuerdo con Castro (2017),

[...] los estudiantes están a la espera de un cambio de actitud pedagógica que enfrente los problemas de maneras más creativas que el señalamiento y la marginalidad, y se la adjudico a los maestros, puesto que en nuestro bagaje teórico y profesional somos quienes tenemos las herramientas epistemológicas para liderar el cambio. (p. 47). 


\section{Financiación}

Fondo Nacional de Desenvolvimiento de la Educación (FNDE), Brasil.

\section{Referencias}

Almeida e Silva, M.P.L. y Evaristo, M.M. (2009). Prefácio: uma reflexão sobre a formação para a qualidade na educação. En A.I.L.F. Dalben y M.F.C. Gomes (org.), Formação continuada de docentes da educação básica (LASEB): construindo parcerias, (pp.13-15). Belo Horizonte: Autêntica Editora.

Castro, A. (2017). La comunicación: una apuesta por la construcción de una escuela reflexiva capaz de criticarse a sí misma. Nodos y Nudos, 42, 39-49.

Gomes, M.F.C., Dalben, A.I. de F., Rocha, A.M. de C. y Alves, M.E. (2009). Eixos metodológicos, estrutura curricular e dinâmica de funcionamento de 12 turmas formadas pelo laseb. En A.I.L.F. Dalben y M.F.C. Gomes (org.), Formação continuada de docentes da educação básica (laseb): construindo parcerias, (pp.19-30). Belo Horizonte: Autêntica Editora.

Dalben, A.I.L.F. y Gomes, M.F.C. (2012). Prefácio. En M. das G. de C. Bregunci (org.). Formação continuada de docentes da educação básica (LASEB): Impactos dos Planos de Ação nas Escolas (pp. 9-14). Belo Horizonte: Autêntica Editora.

Dalben, A.I.L.F (2009). Formação continuada de professores em nivel de especialização: um investimento público que vale a pena? En A.I.L.F. Dalben y M.F.C. Gomes (org.), Formação continuada de docentes da educação básica (LASEB): construindo parcerias (pp. 315-329). Belo Horizonte: Autêntica Editora,

Freire, P. (1996). Pedagogia da Autonomia: saberes necessários à prática educativa. São Paulo: Paz e Terra.

Perrenoud, P. (1999). Formar professores em contextos sociais em mudança: prática reflexiva e participação crítica. Revista Brasileira de Educação, 12, 1-21.

Pino, A. (1995) Semiótica e Cognição na Perspectiva Histórico-cultural. Temas em Psicologia, 2, 31-40.

PBH/SMED (2005). Documento Preliminar para o Curso de Formação Continuada de docentes da educação básica. Belo Horizonte, Secretaria Municipal de Educação, Prefeitura de Belo Horizonte.

Vygotsky, L.S. (1993). Obras escogidas. Tomo II. Madrid: Visor-Aprendizaje.

Vygotsky, L.S. (1995). Obras escogidas. Tomo III. Madrid: Visor-Aprendizaje. 


\section{DIÁLOGO DEL CONOCIMIENTO}

\section{Alianza gobierno, academia y escuela}

Se nos presenta una experiencia brasileña de formación continua, o en ejercicio, de maestros en la educación básica, en donde se combinan los elementos que deben estar presentes en toda política educativa. Están los actores que deben estar: maestros, responsables de la formación básica; el estamento gubernamental, que tiene los recursos para la ejecución de política de formación, y por supuesto, la academia, con sus reflexiones y saberes en pedagogía y educación. Cuando estos trabajan cada uno por su lado, o solo establecen alianzas entre dos de ellos, siempre nos queda la sensación de que algo falta en estos procesos. Quizá, lo más usual es dejar por fuera a los maestros porque los otros -academia y gobierno- saben qué es lo que necesitan aquellos. Sin embargo, también hay que decirlo, cuando los tres construyen una alianza formativa, tampoco significa que resuelven todo, pero los aprendizajes pueden ser más interesantes y pertinentes.

En este caso particular, se trata de un convenio entre Facultad de Educación de la Universidad Federal de Minas Gerais, Secretaría Municipal de Educación de la Alcaldia de Belo Horizonte y maestros de la red municipal de educación de Belo Horizonte, Minas Gerais (Brasil). Como punto de partida, hay que suponer que cada actor tiene una intencionalidad particular, surgida de una lectura del contexto y de unas condiciones que este impone, que ya es dificil de por sí tramitar. Pero entonces aparece una formulación que se repite con tanta frecuencia al punto que nos hemos acostumbrado a usarla en los planes educativos, porque tiene apariencia de acuerdo, o porque es una manera práctica de echar a andar un proceso. Declaramos, entonces, que se trata de "mejorar los procesos de enseñanza y aprendizaje"; pero el significado para cada actor puede ser distinto, su contenido, sus puntos de referencia y, por supuesto, su intencionalidad. Y el proyecto en gran medida se trata de eso, de dotar de contenido dicha expresión, aunque se parta de ideas preliminares, de buscar encuentros en su finalidad y de llevar a cabo las reflexiones que se encuentran en el camino.

Cuando se formula que se pretende construir un proceso "considerando las discusiones socioculturales de la contemporaneidad aliadas a intencionalidades pedagógicas de los profesores y necesidades educativas de los nuevos alumnos", se ubica la materia prima para el estudio, la búsqueda, las discusiones, las posibles respuestas y soluciones prácticas a este planteamiento. Lo esperable, entonces, es dar cuenta de los avances en este diálogo, es decir, de los acercamientos comprensivos de lo que se hace y se quiere hacer con la escuela y la enseñanza contemporáneas. 
Un primer asunto por resaltar en este proyecto es mantenerse en el tiempo; estas iniciativas suelen ser contingentes, aparecen y desaparecen por lo general según los cambios de gobierno o funcionarios. Llevar ocho años en este proceso es interesante y meritorio. Hay, en esta propuesta, un elemento adicional: involucrar a los directivos de las escuelas, que pocas veces sucede. Estos no pueden dejar de considerarse profesores -responsables de la enseñanza-, de lo contrario es ubicarlos en funciones especializadas. Ahora, también es importante considerar a los profesores responsables de la conducción de la institución, lo cual está implícito en la pregunta por lo que es necesario hacer en la escuela y la enseñanza hoy. Así, ya estarian incluidos institución, sujetos y saberes escolares y quizá de esta manera se gana en pertinencia; ver el conjunto tiene ventajas respecto a ver solo las partes. Aunque en este caso se trata de apenas un subconjunto. De hecho, una de las conclusiones que deja el proyecto es que los maestros "pierden la noción de conjunto", y quedan atrapados en problemas concretos de la práctica y no comprenden el "acto educativo como acto político". Pero ya habíamos dicho que todo no se resuelve en un proyecto.

Raúl Barrantes 